Supporting Information for

\title{
Resonance Raman Spectroscopy and Density Functional Theory Calculations on Ferrous Porphyrin Dioxygen Adducts with Different Axial Ligands: Correlation of Ground State Wave Function and Geometric Parameters with Experimental Vibrational Frequencies
}

Asmita Singha, Pradip Kumar Das, Abhishek Dey*

School of Chemical Science, Indian Association for the Cultivation of Science, Kolkata, India 700032 
1. Results:

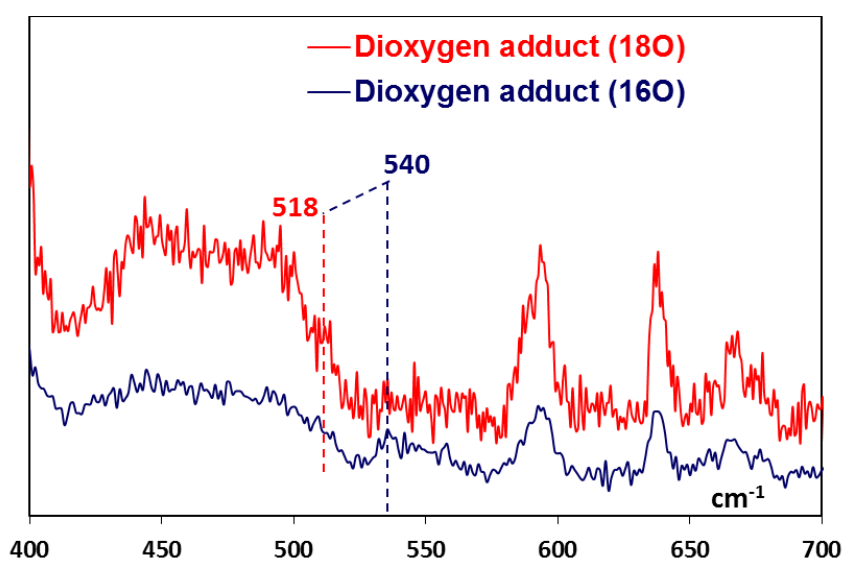

Figure S1: Resonance Raman data of dioxygen adduct of FePPSR-yne in the lower frequency region recorded at 77K. The blue spectra represents 1602 adduct of ferric porphyrin and the red spectra represents the rR data of 1802 substituted ferric superoxide complex.

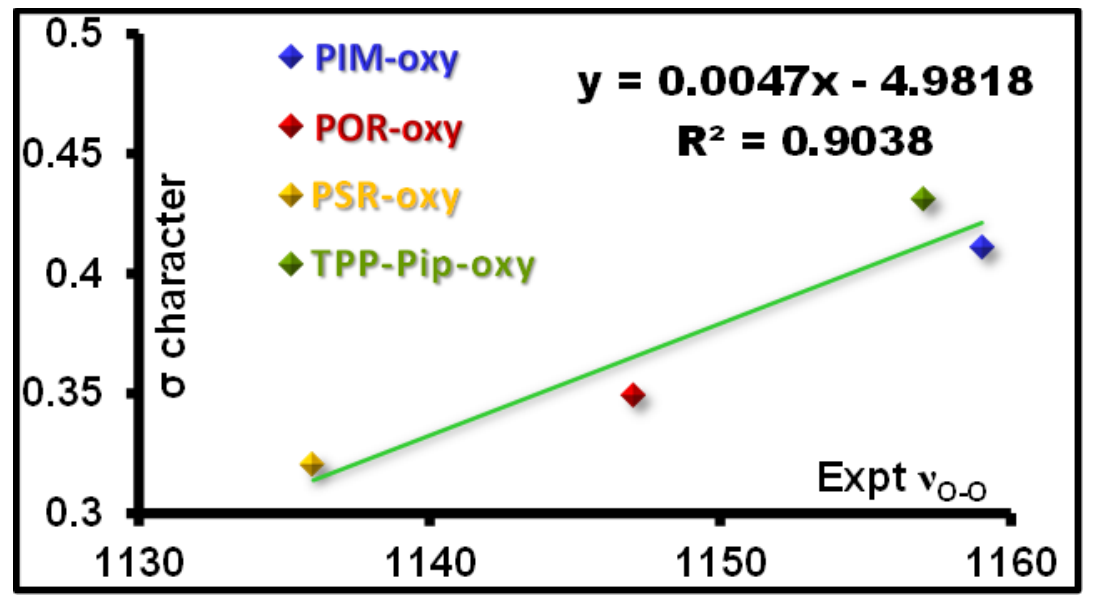

Figure S2: Correlation between calculated $\sigma$ charcter and experimentally observed OO stretching frequency of superoxide complex of PIM, POR, PSR and FeTPP-Pip.

\begin{tabular}{|l|l|l|l|l|}
\hline \multicolumn{5}{|l|}{ Table S1: Calculated coefficient of 3d orbital of Fe in unoccupied molecular orbitals } \\
\hline complexes & $d_{x}{ }^{2-}{ }^{2}$ & $d_{z}{ }^{2}$ & $\beta$ \\
\cline { 2 - 5 } & $\alpha$ & $\beta$ & $\alpha$ & 56.3 \\
\hline PIM & 63.54 & 63.91 & 61.42 & 56.7 \\
\hline $\mathrm{HbO}_{2}$ & 68.1 & 63.6 & 61.3 & \\
\hline
\end{tabular}




\begin{tabular}{|c|c|c|c|c|c|c|c|c|c|c|c|c|c|c|c|c|}
\hline $\begin{array}{l}\mathrm{Fe} \\
\text { porphyrin } \\
\text { complexes }\end{array}$ & PIM & POR & PSR & $\begin{array}{l}\text { FeTPP } \\
\text {-Pip }\end{array}$ & PIM & POR & PSR & $\begin{array}{l}\text { FeTPP } \\
\text {-Pip }\end{array}$ & PIM & POR & PSR & $\begin{array}{l}\text { FeTPP } \\
\text {-Pip }\end{array}$ & PIM & POR & PSR & $\begin{array}{l}\text { FeTPP } \\
\text {-Pip }\end{array}$ \\
\hline $\mathrm{Fe}$ & 55.0 & 42.9 & 39.9 & 54.8 & 10.9 & 14.8 & 10.9 & 10.8 & 61.4 & 57.2 & 51.8 & 61.5 & 56.3 & 49.2 & 43.5 & 55.2 \\
\hline $\mathrm{O}_{2}$ & 25.8 & 21.4 & 17.3 & 23.8 & 85.5 & 79.9 & 85 & 82.8 & 16.8 & 7.2 & 10.9 & 18.1 & 22.5 & 15.9 & 9.8 & 25 \\
\hline $\mathrm{N}$ (Py rrole) & 0.5 & 1.1 & 0 & 0.5 & 0.88 & 0.5 & 0 & 0.8 & 4.6 & 15.7 & 9.3 & 6.4 & 16.5 & 8.9 & 12.9 & 6.8 \\
\hline $\begin{array}{l}\text { X } \\
\text { group of } \\
\text { axial ligand) }\end{array}$ & 0.0 & 10.0 & 12.8 & 0.0 & 0.0 & 1.7 & 1.1 & 0.0 & 0.2 & 7.6 & 2.6 & 6.0 & 0.2 & 11.2 & 3.0 & 6.3 \\
\hline
\end{tabular}

\begin{tabular}{|c|c|c|c|c|c|c|}
\hline \multicolumn{7}{|c|}{$\begin{array}{l}\text { Table S3: DFT calculated and experimentally observed stretching frequencies of } \mathrm{Fe}-\mathrm{O} \text { and } \mathrm{O}-\mathrm{O} \text { of superoxide } \\
\text { complexes of Fe porphyrins }\end{array}$} \\
\hline $\begin{array}{l}\text { Porphyrin } \\
\text { complexes }\end{array}$ & $\begin{array}{l}\text { Calculated } \\
v(\mathrm{Fe}-\mathrm{O})\left(\mathrm{cm}^{-1}\right)\end{array}$ & $\begin{array}{l}\text { Calculated } \\
v(\mathrm{O}-\mathrm{O})\left(\mathrm{cm}^{-1}\right)\end{array}$ & $\begin{array}{l}\text { experimental } \\
v(\mathrm{Fe}-\mathrm{O})\left(\mathrm{cm}^{-1}\right)\end{array}$ & $\begin{array}{l}\text { experimenta } \\
1 \quad v(\mathrm{O}-\mathrm{O}) \\
\left(\mathrm{cm}^{-1}\right)\end{array}$ & $\begin{array}{l}\text { Ratio of calc. } \\
\text { and expt. } \\
v(\mathrm{O}-\mathrm{O}) \quad\left(\mathrm{cm}^{-}\right. \\
\left.{ }^{1}\right)\end{array}$ & $\begin{array}{l}\text { Ratio of calc. } \\
\text { and expt. } \\
v(\mathrm{Fe}-\mathrm{O}) \quad\left(\mathrm{cm}^{-}\right. \\
\left.{ }^{1}\right)\end{array}$ \\
\hline PIM & 557 & 1230 & 584 & 1159 & 0.94 & 1.05 \\
\hline POR & 529 & 1211 & 570 & 1147 & 0.94 & 1.08 \\
\hline PSR & 511 & 1208 & 541 & 1136 & 0.94 & 1.06 \\
\hline $\begin{array}{l}\text { FeTPP- } \\
\text { Pip }\end{array}$ & 550 & 1233 & 575 & 1157 & 0.94 & 1.04 \\
\hline
\end{tabular}

2. DFT optimised coordinates:

\subsection{Superoxide complex of PIM:}

$\begin{array}{llll}\text { C } & -3.35421900 & 3.24978100 & -0.15887400 \\ C & -4.14657500 & 2.19086300 & 0.19071700 \\ C & -3.35041400 & 0.99440500 & 0.01102200 \\ C & -2.05435800 & 2.70897400 & -0.49913500 \\ \text { C } & -0.94145600 & 3.50116500 & -0.83953700\end{array}$




$$
-3.85329400
$$$$
-0.30866100
$$

0.17402100

$-3.10745200$

$-1.46191700$

$-3.68106200$

$-2.79075600$

$-2.69451800$

$-3.63430300$

$-1.50664000$

$-2.82835700$

$-0.25605100$

0.90442900

2.23044800

3.10685300

2.31595100

2.85809200

2.06515000

2.59746900

1.52887400

0.34555500

$-2.75876700$

$-4.71697200$

$-5.18384700$

$-3.61457800$

1.53101900

3.65215800

4. 18969900

2.45290300

$-1.77633100$

$-2.06325000$

0.68598500

0.96909000

$-1.11898800$
$-3.35272500$

$-2.56190600$

$-3.10814900$

$-2.05650000$

$-0.86379900$

0.43281600

1.59106200

2.92990200

3.78346800

2.96811300

$-4.70563200$

$-3.02911100$

0.06529300

2.20709400

0.52190600

4.30716500

$-0.17925300$

4.87129700

$-1.28210300$

3.16900700

$-1.41823000$

$-2.07085300$

$-1.57605900$

$-4.16564300$

$-1.62835800$

$-1.49431900$

$-0.52196400$

1.32338400

$-0.39440500$

$1.62397900-1.00224600$

$-1.18279700$

$-1.12985100$

4. 98481300

$-0.96208000$ 
$-1.41061200$

$-0.98663900$

$-1.57098300$

$-1.14660000$

$-1.44013900$

$-1.79230700$

$-1.04557100$

$-1.56484300$

$-5.27204900$

$-6.35251800$

$-5.55702800$

$-7.67820600$

$-6.88295000$

$-7.94801100$

$-8.50290000$

$-7.08392400$

$-8.98325300$

$-0.13618800$

0.19929600

$-0.34254600$

0.32195800

$-0.21988900$

0.11286100

0.57662000

$-0.37963500$

0.20856800

$-0.53923400$

4.35385900
5.78535400

0.16471800

5.61433600

7.17388500

0.03721500

7.00294000

7.78714400

7.77870200

7.47223700

8.87126800

$-0.48528800$

0.62576300

$-0.08764600$

$-0.19334400$

$-1.06950600$

1.87995400

$-0.26302100$

$-1.24491800$

2.30611100

$-0.84101800$

1.48437800

0.04677800

$-0.41913400$

$-1.69548100$

3.28495900

$-0.97821600$

1.81651700

$-4.82197100$

$-1.44357600$

$-5.28575800$

$-2.73596000$

$-5.77424600$

$-0.42020700$

$-6.65938100$

$-2.99596300$

$-7.14791400$

$-0.68041400$

$-7.59524300$

$-1.96959100$

$-6.99897900$

$-4.00637600$

$-7.87034600$

0.12832600

$-8.66769200$

$-2.17320700$

0.07239300

$-0.79774700$

0.58103200

$-1.28776700$ 
$\begin{array}{llll}5.09981500 & 0.64377000 & -0.07483600\end{array}$

$5.04455500 \quad 0.63661400 \quad-2.51148100$

C

6.50654100

0.74663600

$-0.10735500$

C

6.44427900

0.74512300

$-2.54836700$

C

7.16495600

0.79503700

$-1.34446600$

$\mathrm{H}$

7.05271100

0.78009400

0.83695000

$\mathrm{H}$

6.96554000

0.78686500

$-3.51083500$

H

8.25786300

0.87515600

$-1.36172700$

$\mathrm{N}$

4.36010400

0.60364500

1.12627500

C

4.79528200

0.52141000

2.43716800

o

5.98020400

0.49201500

2.78884000

C

3.64116600

0.41425900

3.44274100

C

3.52338200

$-1.02795900$

3.99104300

C

$\mathrm{H}$

2.32896900

$-1.23811100$

4.94965900

4.46836200

$-1.26619700$

4.51069600

C

1.01853400

$-1.69107900$

4.27354600

$\mathrm{H}$

1.17316000

$-2.66182200$

3.77118600

$\mathrm{N}$

0.49889700

$-0.77454700$

3.24846900

$\mathrm{H}$

0.22946800

$-1.83782400$

5.03371100

$\mathrm{H}$

3.34741400

0.59678800

0.98084500

$\mathrm{H}$

3.86815300

1.10043900

4.27895300

$\mathrm{H}$

2.68553400

0.74512900

2.99731700

$\mathrm{H}$

3.44970400

$-1.74104600$

3.14582800

H

$-1.50203700$

5.31029200

1.14800600

$\mathrm{H}$

$-0.76516700$

5.00101200

$-3.10047600$

$\mathrm{H}$

$-6.14300600$

0.35065600

$-1.17530000$

$\mathrm{H}$

$-4.72601300$

$-1.38012400$

2.52324200

H

0.35327600

$-4.55637900$

$-3.53878200$ 


$\begin{array}{llcc}\mathrm{H} & -0.59441600 & -5.42691500 & 0.58828000 \\ \mathrm{H} & 4.46564800 & 0.58873900 & -3.44041900 \\ \mathrm{H} & 2.57334100 & -2.02794700 & 5.68556900 \\ \mathrm{H} & 2.14852900 & -0.31937700 & 5.54104600 \\ \mathrm{C} & 0.42127800 & -1.02989200 & 1.90696100 \\ \mathrm{~N} & -0.09323700 & 0.01251900 & 1.24745000 \\ \mathrm{C} & -0.35463600 & 0.98107600 & 2.20293500 \\ \mathrm{C} & 0.00566400 & 0.50888100 & 3.44899700 \\ \mathrm{H} & 0.72397100 & -1.97300500 & 1.45571500 \\ \mathrm{H} & -0.79034900 & 1.93885400 & 1.92765500 \\ \mathrm{H} & -0.04543100 & 0.95646600 & 4.43933000 \\ \mathrm{O} & -1.00474400 & 0.11028000 & -2.52732900 \\ \mathrm{O} & -0.09826200 & 0.21667000 & -3.43752400\end{array}$

\subsection{Superoxide complex of POR}

$\begin{array}{llrl}\text { C } & 4.78332400 & -2.04181300 & 0.54154700 \\ \text { C } & 5.33720700 & -0.79101700 & 0.45809900 \\ \text { C } & 4.27419400 & 0.10855100 & 0.05781600 \\ \text { C } & 3.39630500 & -1.91431000 & 0.14182400 \\ \text { C } & 2.49730300 & -2.99608500 & 0.02280800 \\ \text { C } & 4.40988100 & 1.51188000 & 0.03513600 \\ \text { C } & 3.31743700 & 2.38628200 & -0.09230000 \\ \text { C } & 3.40731600 & 3.82499500 & 0.08065300 \\ \text { C } & 2.13195400 & 4.31054400 & 0.00207200 \\ \text { C } & 1.26886200 & 3.18002700 & -0.29238700 \\ \text { C } & -0.10414700 & 3.29548700 & -0.57825500 \\ \text { C } & -0.89337400 & 2.21928800 & -1.03728500\end{array}$




\begin{tabular}{|c|c|c|c|}
\hline C & -2.19054200 & 2.38882400 & -1.65980900 \\
\hline $\mathrm{C}$ & -2.55087200 & 1.16592200 & -2.16722600 \\
\hline C & -1.50291500 & 0.23745800 & -1.79635000 \\
\hline $\mathrm{C}$ & -1.54833800 & -1.15418000 & -2.03059000 \\
\hline C & -0.58445200 & -2.03742100 & -1.50452600 \\
\hline C & -0.72214700 & -3.48144900 & -1.53135800 \\
\hline $\mathrm{C}$ & 0.36989200 & -3.99255900 & -0.88675300 \\
\hline $\mathrm{C}$ & 1.19562900 & -2.86388900 & -0.49710500 \\
\hline $\mathrm{H}$ & 1.79898700 & 5.34228800 & 0.11016800 \\
\hline $\mathrm{H}$ & 4.32822000 & 4.37862800 & 0.25983900 \\
\hline $\mathrm{H}$ & 6.35756800 & -0.48905800 & 0.69242100 \\
\hline $\mathrm{H}$ & 5.26509500 & -2.96997600 & 0.84752300 \\
\hline $\mathrm{H}$ & 0.62039400 & -5.03859300 & -0.71382100 \\
\hline $\mathrm{H}$ & -1.56176400 & -4.01856200 & -1.97168400 \\
\hline $\mathrm{H}$ & -3.44059300 & 0.91114200 & -2.74283800 \\
\hline $\mathrm{H}$ & -2.72258400 & 3.33607800 & -1.74708000 \\
\hline $\mathrm{N}$ & 2.00704700 & 2.00877200 & -0.33326500 \\
\hline $\mathrm{N}$ & 3.09978500 & -0.59094500 & -0.13304100 \\
\hline $\mathrm{N}$ & 0.58566500 & -1.67272800 & -0.85753100 \\
\hline N & -0.48967200 & 0.89888400 & -1.12046300 \\
\hline C & 2.95532400 & -4.36618200 & 0.41379900 \\
\hline C & 2.30293400 & -5.05571900 & 1.46221900 \\
\hline $\mathrm{C}$ & 4.02410600 & -5.01161900 & -0.24903300 \\
\hline $\mathrm{C}$ & 2.71198000 & -6.34414300 & 1.83944500 \\
\hline C & 4.43391600 & -6.29961400 & 0.13000500 \\
\hline C & 3.78030700 & -6.97120100 & 1.17669800 \\
\hline $\mathrm{H}$ & 2.19467900 & -6.85818800 & 2.65842500 \\
\hline $\mathrm{H}$ & 5.26173400 & -6.78383000 & -0.402196 \\
\hline
\end{tabular}




\begin{tabular}{|c|c|c|c|}
\hline $\mathrm{H}$ & 4.09945900 & -7.97802300 & 1.47146700 \\
\hline C & 5.78314400 & 2.08125600 & 0.21449000 \\
\hline C & 6.76262400 & 1.89049600 & -0.78654500 \\
\hline C & 6.14428200 & 2.80033200 & 1.37629000 \\
\hline C & 8.05831200 & 2.40851100 & -0.63457000 \\
\hline C & 7.43932300 & 3.32119000 & 1.52734400 \\
\hline C & 8.40158900 & 3.12806500 & 0.52221700 \\
\hline $\mathrm{H}$ & 8.80121300 & 2.25321300 & -1.42629700 \\
\hline $\mathrm{H}$ & 7.69944700 & 3.87246400 & 2.43913100 \\
\hline $\mathrm{H}$ & 9.41351000 & 3.53349300 & 0.64116100 \\
\hline C & -0.75582000 & 4.64058600 & -0.48389600 \\
\hline C & -1.78634300 & 4.86716800 & 0.45655900 \\
\hline C & -0.38270600 & 5.70745700 & -1.33327000 \\
\hline C & -2.41646400 & 6.11767600 & 0.55235800 \\
\hline C & -1.01098300 & 6.95912400 & -1.23776700 \\
\hline C & -2.02987000 & 7.17022400 & -0.29376900 \\
\hline $\mathrm{H}$ & -3.20854200 & 6.27221800 & 1.29507900 \\
\hline $\mathrm{H}$ & -0.70837500 & 7.77053400 & -1.91065700 \\
\hline $\mathrm{H}$ & -2.52082700 & 8.14784800 & -0.21967200 \\
\hline $\mathrm{Fe}$ & 1.31061700 & 0.16349400 & -0.60240500 \\
\hline C & -2.61699200 & -1.72925000 & -2.91864300 \\
\hline C & -4.00085300 & -1.76877100 & -2.57307800 \\
\hline C & -2.22988600 & -2.26540500 & -4.16532100 \\
\hline C & -4.94413200 & -2.31288100 & -3.47359100 \\
\hline C & -3.16319700 & -2.80858900 & -5.05971200 \\
\hline C & -4.52178400 & -2.82330700 & -4.70786900 \\
\hline $\mathrm{H}$ & -5.99614100 & -2.31367300 & -3.18498500 \\
\hline $\mathrm{H}$ & -2.83017100 & -3.20912100 & -6.023992 \\
\hline
\end{tabular}




\begin{tabular}{|c|c|c|c|}
\hline $\mathrm{H}$ & -5.26744900 & -3.24022900 & -5.39549700 \\
\hline $\mathrm{N}$ & -4.38439500 & -1.26035900 & -1.31304900 \\
\hline C & -5.64215100 & -0.86645800 & -0.88149700 \\
\hline O & -6.68994100 & -1.04579900 & -1.51617700 \\
\hline C & -5.59841800 & -0.15949900 & 0.47982600 \\
\hline C & -6.92665700 & 0.53340700 & 0.83772500 \\
\hline C & -6.78863100 & 1.66403300 & 1.87955300 \\
\hline $\mathrm{C}$ & -6.55913900 & 1.22565500 & 3.34076200 \\
\hline $\mathrm{H}$ & -6.46877600 & 2.11460500 & 3.99367900 \\
\hline $\mathrm{H}$ & -7.40550400 & 0.61887400 & 3.70544900 \\
\hline $\mathrm{H}$ & -3.59480600 & -0.98829100 & -0.71951300 \\
\hline $\mathrm{H}$ & -5.31369400 & -0.90021900 & 1.24987300 \\
\hline $\mathrm{H}$ & -4.77687900 & 0.58349300 & 0.45242400 \\
\hline $\mathrm{H}$ & -7.35308100 & 0.95934000 & -0.08845100 \\
\hline $\mathrm{H}$ & 1.47415400 & -4.56132900 & 1.98151300 \\
\hline $\mathrm{H}$ & 4.52332100 & -4.49560100 & -1.07662100 \\
\hline $\mathrm{H}$ & 6.48853700 & 1.33638000 & -1.69094300 \\
\hline $\mathrm{H}$ & 5.39901500 & 2.93658300 & 2.16774300 \\
\hline $\mathrm{H}$ & -2.07894800 & 4.04767000 & 1.12220000 \\
\hline $\mathrm{H}$ & 0.40105000 & 5.53900700 & -2.07987500 \\
\hline $\mathrm{H}$ & -1.16663200 & -2.23353500 & -4.42665000 \\
\hline $\mathrm{H}$ & -7.71529400 & 2.27190000 & 1.88277900 \\
\hline $\mathrm{H}$ & -5.97590800 & 2.35173800 & 1.57165400 \\
\hline 0 & 0.90266100 & 0.19772500 & 1.28518500 \\
\hline $\mathrm{C}$ & 0.14025300 & -0.53091300 & 2.08283100 \\
\hline $\mathrm{C}$ & -1.23313400 & -0.22546900 & 2.30325700 \\
\hline $\mathrm{C}$ & -2.01068100 & -0.94782300 & 3.23264300 \\
\hline C & -1.44080000 & -2.01967600 & 3.95505500 \\
\hline
\end{tabular}




$\begin{array}{llcl}\text { C } & -0.08626500 & -2.33001000 & 3.75250100 \\ \mathrm{C} & 0.69580200 & -1.60626000 & 2.84418200 \\ \mathrm{C} & -3.42461300 & -0.59656200 & 3.44011600 \\ \mathrm{C} & -4.07170700 & 0.62704200 & 3.26151700 \\ \mathrm{~N} & -5.38654600 & 0.37515600 & 3.53053500 \\ \mathrm{~N} & -5.56302500 & -0.94047300 & 3.87336400 \\ \mathrm{~N} & -4.37994100 & -1.51914500 & 3.82801300 \\ \mathrm{H} & -3.70630000 & 1.61172300 & 2.97891100 \\ \mathrm{H} & -2.05755600 & -2.57976500 & 4.66446100 \\ \mathrm{H} & 0.37173000 & -3.14696400 & 4.32643900 \\ \mathrm{H} & 1.75606400 & -1.83993500 & 2.70029100 \\ \mathrm{H} & -1.67311900 & 0.59213800 & 1.72029400 \\ \mathrm{H} & -7.65990100 & -0.21770400 & 1.18687900 \\ \mathrm{O} & 1.74775300 & 0.13817900 & -2.37259500 \\ \mathrm{O} & 2.83612900 & 0.71386200 & -2.79083200\end{array}$

\subsection{Superoxide complex of PSR}

$\begin{array}{llll}\text { C } & -3.61635800 & 2.87230200 & -0.12241900 \\ \text { C } & -4.24189800 & 1.73341500 & 0.31045800 \\ \text { C } & -3.28857100 & 0.65145700 & 0.17073400 \\ \text { C } & -2.25661000 & 2.49918200 & -0.45684200 \\ \text { C } & -1.25225700 & 3.41810200 & -0.81816000 \\ \text { C } & -3.59391600 & -0.70497400 & 0.39878200 \\ \text { C } & -2.73026600 & -1.76178900 & 0.05038600 \\ \text { C } & -3.13131900 & -3.15510700 & 0.02848600 \\ \text { C } & -2.09411900 & -3.85836600 & -0.52304200 \\ \text { C } & -1.04341000 & -2.89983300 & -0.80870300\end{array}$


$3.44535200-1.58591000-1.34738100$

$-4.02522000$

3.87970700

$-0.19157400$

1.06888800

5.08459100

$-1.11353600$

3.37822900

3.64820900

$-0.95700300$

4.52538800

$-1.46149100$

$-1.42969700$

3.04288300

$-3.70585000$

$-1.85426300$

$-1.44469900$

$-1.62210200$

$-0.44855100$

$-2.07400900$

1.13721000

$-0.28418500$

0.59093400

1.76072300

$-0.84985600$

1.21840200

$-1.00121100$

$-1.02014100$

$-1.61285000$

4.85724700

$-1.01263300$

$-2.07531300$

5.65903700

0.05564500

$-1.48072500$

5.45238400

$-2.28815000$

$-2.40342400$

7.00885100

$-0.14652100$

$-1.80631200$

6.80274400

$-2.48997000$

$-2.27108700$

7.58639200

$-1.42047200$ 


\begin{tabular}{|c|c|c|c|}
\hline $\mathrm{H}$ & -2.75522600 & 7.61411100 & 0.69781000 \\
\hline $\mathrm{H}$ & -1.70131900 & 7.24274500 & -3.48912200 \\
\hline $\mathrm{H}$ & -2.52617500 & 8.64125100 & -1.57798500 \\
\hline C & -4.93484200 & -1.06056400 & 0.96471600 \\
\hline C & -6.12766200 & -0.84567900 & 0.23764700 \\
\hline $\mathrm{C}$ & -5.03125800 & -1.64420900 & 2.24834200 \\
\hline $\mathrm{C}$ & -7.37520100 & -1.19389300 & 0.77954400 \\
\hline$C$ & -6.27783300 & -1.99499000 & 2.79003400 \\
\hline C & -7.45570500 & -1.76989900 & 2.05837100 \\
\hline $\mathrm{H}$ & -8.28746900 & -1.02187700 & 0.19545000 \\
\hline $\mathrm{H}$ & -6.32854200 & -2.44144000 & 3.79051700 \\
\hline $\mathrm{H}$ & -8.42993100 & -2.04307200 & 2.48116000 \\
\hline $\mathrm{C}$ & 0.46974200 & -4.68521800 & -1.66882300 \\
\hline C & 1.34344200 & -5.52472200 & -0.94018300 \\
\hline C & -0.20089900 & -5.23153400 & -2.78735100 \\
\hline $\mathrm{C}$ & 1.54634300 & -6.86032000 & -1.32176900 \\
\hline C & -0.00094000 & -6.56762800 & -3.16775700 \\
\hline $\mathrm{C}$ & 0.87550600 & -7.38775500 & -2.43767400 \\
\hline $\mathrm{H}$ & 2.22580000 & -7.49369400 & -0.73858700 \\
\hline $\mathrm{H}$ & -0.52844100 & -6.96744600 & -4.04226100 \\
\hline $\mathrm{H}$ & 1.03346500 & -8.43132100 & -2.73512300 \\
\hline $\mathrm{Fe}$ & -0.42336300 & 0.07588000 & -0.63968300 \\
\hline C & 4.33690800 & 1.13472700 & -0.61961900 \\
\hline C & 4.81687000 & 1.11704000 & 0.72634000 \\
\hline C & 5.23306200 & 1.43418600 & -1.66012400 \\
\hline $\mathrm{C}$ & 6.17883600 & 1.39580400 & 0.98622200 \\
\hline C & 6.58619800 & 1.71089800 & -1.40167200 \\
\hline C & 7.04678100 & 1.68743000 & -0.07573600 \\
\hline
\end{tabular}




\begin{tabular}{|c|c|c|c|}
\hline $\mathrm{H}$ & 6.52224500 & 1.37262800 & 2.02190000 \\
\hline $\mathrm{H}$ & 7.26930200 & 1.94130200 & -2.22748300 \\
\hline $\mathrm{H}$ & 8.10019300 & 1.90130200 & 0.14371200 \\
\hline $\mathrm{N}$ & 3.89204100 & 0.81743400 & 1.74419200 \\
\hline C & 4.11333500 & 0.62961400 & 3.10443000 \\
\hline O & 5.20765900 & 0.78387800 & 3.66542400 \\
\hline C & 2.84801700 & 0.16651000 & 3.82733400 \\
\hline C & 2.55871300 & -1.34649100 & 3.59188100 \\
\hline C & 1.05718900 & -1.71695700 & 3.61041900 \\
\hline $\mathrm{H}$ & 3.10675100 & -1.93142900 & 4.35506000 \\
\hline C & 0.34523900 & -1.58559400 & 2.24794100 \\
\hline $\mathrm{H}$ & 0.90080400 & -2.17428900 & 1.49332000 \\
\hline S & 0.16909600 & 0.14860100 & 1.63103100 \\
\hline $\mathrm{H}$ & -0.66431400 & -2.03129600 & 2.32041700 \\
\hline $\mathrm{H}$ & 2.91625000 & 0.67425900 & 1.43381300 \\
\hline $\mathrm{H}$ & 2.98239700 & 0.37382400 & 4.90244900 \\
\hline $\mathrm{H}$ & 1.98315600 & 0.74963700 & 3.45942400 \\
\hline $\mathrm{H}$ & 2.97640100 & -1.65770700 & 2.61435200 \\
\hline $\mathrm{H}$ & -2.16279500 & 5.21321900 & 1.05261000 \\
\hline $\mathrm{H}$ & -1.12776900 & 4.83490200 & -3.12131200 \\
\hline $\mathrm{H}$ & -6.06408300 & -0.41070100 & -0.76591100 \\
\hline $\mathrm{H}$ & -4.11246900 & -1.81223900 & 2.82077600 \\
\hline $\mathrm{H}$ & 1.85584900 & -5.11967200 & -0.06062200 \\
\hline $\mathrm{H}$ & -0.87755300 & -4.58822500 & -3.36051100 \\
\hline $\mathrm{H}$ & 4.84892300 & 1.44412200 & -2.68667900 \\
\hline $\mathrm{H}$ & 0.95908800 & -2.77677600 & 3.92687500 \\
\hline $\mathrm{H}$ & 0.52601000 & -1.11536300 & 4.37489400 \\
\hline O & -0.81536800 & 0.00375700 & -2.44599900 \\
\hline
\end{tabular}


2.4 Superoxide complex of FeTPP(PIP)

\begin{tabular}{|c|c|c|c|}
\hline $\mathrm{Fe}$ & 3.56789100 & 9.45032600 & 6.24907200 \\
\hline $\mathrm{N}$ & 5.42686000 & 9.68496900 & 5.52235100 \\
\hline $\mathrm{N}$ & 2.79792400 & 9.86701700 & 4.46492300 \\
\hline N & 1.73622500 & 9.28973200 & 7.00064700 \\
\hline N & 4.35305600 & 9.10990700 & 8.06440200 \\
\hline C & 6.76715800 & 9.52082100 & 7.61138400 \\
\hline C & 4.88649300 & 9.91254200 & 3.10361500 \\
\hline $\mathrm{C}$ & 3.48882900 & 9.98511900 & 3.26549500 \\
\hline C & 1.46205900 & 10.09568000 & 4.14399000 \\
\hline C & 7.22032900 & 9.90047300 & 4.05032400 \\
\hline C & 7.74116400 & 9.87766700 & 5.31544900 \\
\hline C & 6.62628400 & 9.70516500 & 6.22353800 \\
\hline C & 5.78153300 & 9.81003800 & 4.18502400 \\
\hline C & 1.32666800 & 10.40657500 & 2.73863600 \\
\hline C & 2.57806800 & 10.31240400 & 2.19019200 \\
\hline C & 2.28772600 & 8.43094700 & 9.27486900 \\
\hline C & 3.67914800 & 8.61829100 & 9.17572500 \\
\hline C & 1.38975900 & 8.79193200 & 8.25119300 \\
\hline C & 0.38021800 & 10.00865800 & 5.03971800 \\
\hline C & 4.60790000 & 8.36211800 & 10.25682200 \\
\hline C & 5.68570300 & 9.18272800 & 8.44648300 \\
\hline C & 5.84702700 & 8.73845200 & 9.81533100 \\
\hline C & -0.58200000 & 9.26750500 & 7.24323700 \\
\hline C & -0.04848900 & 8.77390800 & 8.40410800 \\
\hline $\mathrm{C}$ & 0.52880500 & 9.57118400 & 6.36958700 \\
\hline
\end{tabular}




\begin{tabular}{|c|c|c|c|}
\hline $\mathrm{H}$ & 7.75490000 & 9.98570800 & 3.10519200 \\
\hline $\mathrm{H}$ & 8.78611100 & 9.94540200 & 5.61439100 \\
\hline $\mathrm{H}$ & 0.39057900 & 10.65691200 & 2.24145700 \\
\hline $\mathrm{H}$ & 2.87274800 & 10.47799300 & 1.15467400 \\
\hline $\mathrm{H}$ & 4.33820800 & 7.94097200 & 11.22435900 \\
\hline $\mathrm{H}$ & 6.79792100 & 8.68148500 & 10.34331900 \\
\hline $\mathrm{H}$ & -1.63197200 & 9.39407800 & 6.98327200 \\
\hline $\mathrm{H}$ & -0.57465900 & 8.43397200 & 9.29526600 \\
\hline C & 8.13336800 & 9.60074700 & 8.22314400 \\
\hline C & 8.44363100 & 10.62732800 & 9.14273700 \\
\hline C & 9.13248700 & 8.65118900 & 7.91216600 \\
\hline C & 9.71697300 & 10.70862500 & 9.72758400 \\
\hline $\mathrm{H}$ & 7.67549000 & 11.36787100 & 9.39280600 \\
\hline C & 10.40589100 & 8.73246600 & 8.49688400 \\
\hline $\mathrm{H}$ & 8.89674700 & 7.83938300 & 7.21532100 \\
\hline C & 10.70314200 & 9.76223500 & 9.40496600 \\
\hline $\mathrm{H}$ & 9.93949700 & 11.51585400 & 10.43488000 \\
\hline $\mathrm{H}$ & 11.16584100 & 7.98326600 & 8.24700700 \\
\hline $\mathrm{H}$ & 11.69755200 & 9.82471500 & 9.86111900 \\
\hline C & -1.00285400 & 10.29946900 & 4.54067300 \\
\hline C & -1.72960200 & 11.40291100 & 5.04030500 \\
\hline C & -1.61643200 & 9.46973600 & 3.57544100 \\
\hline $\mathrm{C}$ & -3.02885200 & 11.67424200 & 4.58378900 \\
\hline $\mathrm{H}$ & -1.26442300 & 12.05231000 & 5.79061700 \\
\hline C & -2.91565600 & 9.74110600 & 3.11905100 \\
\hline C & -3.62563400 & 10.84458100 & 3.62044900 \\
\hline $\mathrm{H}$ & -3.57466700 & 12.53813900 & 4.98019700 \\
\hline 11 & -3.37723600 & 9.08267900 & 2.374312 \\
\hline
\end{tabular}




\begin{tabular}{|c|c|c|c|}
\hline $\mathrm{H}$ & -4.64025800 & 11.05536600 & 3.26418800 \\
\hline C & 1.72292600 & 7.85990600 & 10.54008400 \\
\hline C & 1.09123000 & 6.59592100 & 10.53100500 \\
\hline C & 1.80094600 & 8.56547900 & 11.76177200 \\
\hline C & 0.55837900 & 6.05235100 & 11.71019700 \\
\hline $\mathrm{H}$ & 1.03181000 & 6.03816200 & 9.58988400 \\
\hline $\mathrm{C}$ & 1.26846100 & 8.02118200 & 12.94077200 \\
\hline $\mathrm{H}$ & 2.27704400 & 9.55230600 & 11.77821900 \\
\hline C & 0.64609700 & 6.76226800 & 12.91910200 \\
\hline $\mathrm{H}$ & 0.07829000 & 5.06744800 & 11.68423000 \\
\hline $\mathrm{H}$ & 1.33530700 & 8.58581900 & 13.87787800 \\
\hline $\mathrm{H}$ & 0.23088500 & 6.33687300 & 13.83969100 \\
\hline C & 5.43525000 & 9.99944800 & 1.71110100 \\
\hline C & 5.20048600 & 8.95393200 & 0.79028300 \\
\hline C & 6.17835300 & 11.12177900 & 1.28205200 \\
\hline C & 5.70049800 & 9.02635200 & -0.51918800 \\
\hline $\mathrm{H}$ & 4.62741600 & 8.07858400 & 1.11529500 \\
\hline C & 6.67817700 & 11.19386000 & -0.02763400 \\
\hline $\mathrm{H}$ & 6.35344200 & 11.94539700 & 1.98333100 \\
\hline C & 6.44165100 & 10.14574400 & -0.93220100 \\
\hline $\mathrm{H}$ & 5.51298300 & 8.20267600 & -1.21747600 \\
\hline $\mathrm{H}$ & 7.24833000 & 12.07510400 & -0.34347700 \\
\hline $\mathrm{H}$ & 6.83150500 & 10.20200300 & -1.95479900 \\
\hline O & 3.69065800 & 7.69428200 & 5.90464300 \\
\hline O & 2.70551400 & 7.07644900 & 5.34747000 \\
\hline $\mathrm{H}$ & -1.06846900 & 8.60025800 & 3.19636600 \\
\hline C & 3.30476300 & 11.99241200 & 8.08767500 \\
\hline C & 4.30063700 & 12.49243300 & 5.89957500 \\
\hline
\end{tabular}




\begin{tabular}{|c|c|c|c|}
\hline C & 2.88733200 & 13.46187500 & 8.27332100 \\
\hline $\mathrm{H}$ & 4.30703500 & 11.81656300 & 8.51756100 \\
\hline $\mathrm{H}$ & 2.60567100 & 11.30797700 & 8.59371400 \\
\hline C & 3.91411500 & 13.97652000 & 6.02316000 \\
\hline $\mathrm{H}$ & 5.31376800 & 12.31937000 & 6.30478400 \\
\hline $\mathrm{H}$ & 4.30744000 & 12.16609300 & 4.84775200 \\
\hline C & 3.82244800 & 14.40864100 & 7.49833500 \\
\hline $\mathrm{H}$ & 2.88237100 & 13.70562900 & 9.35274400 \\
\hline $\mathrm{H}$ & 1.84469300 & 13.59230900 & 7.91662200 \\
\hline $\mathrm{H}$ & 4.65392800 & 14.59129700 & 5.47614700 \\
\hline $\mathrm{H}$ & 2.93725000 & 14.14217200 & 5.52367800 \\
\hline $\mathrm{H}$ & 3.47431500 & 15.45522700 & 7.57883800 \\
\hline $\mathrm{H}$ & 4.83381600 & 14.37397200 & 7.95233400 \\
\hline $\mathrm{N}$ & 3.37732000 & 11.58971000 & 6.65090800 \\
\hline $\mathrm{H}$ & 2.43475600 & 11.72614800 & 6.252067 \\
\hline
\end{tabular}

